\title{
In Vivo Comparison of T-PRF, L-PRF and Xenograft on Early Phase Bone Healing
}

\author{
Duymaz Bilge', Dogan Onur Ozen', Soluk Tekkesin Merva², Tunali Mustafa ${ }^{3}$, Ramazanoglu Mustafa', \\ Firatli Erhan ${ }^{4}$ \\ 'Istanbul University, Faculty of Dentistry, Department of Oral and Maxillofacial Surgery \\ ${ }^{2}$ Istanbul University, Institute of Oncology, Department of Tumour Pathology \\ ${ }^{3}$ Bezmialem University, Faculty of Dentistry, Department of Periodontology \\ ${ }^{4}$ Istanbul University, Faculty of Dentistry, Department of Periodontology
}

\section{Background}

One of the important problems of oral and maxillofacial surgery is the augmentation of bone defects. The osteoconductive effects of xenografts can be induced by autogenous materials such as platelet rich fibrins. L-PRF and T-PRF may have an osteoinductive effect with contents. At the same time these materials have a strong fibrin network structure. TPRF method is based on the hypothesis that titanium may be more effective at activating platelets than the silica activators used with glass tubes in L-PRF method.

The use of this platelet concentrates during bone grafting offers the following advantages. First, the fibrin clot plays an important mechanical role, with the PRF membrane maintaining and protecting the grafted biomaterials and PRF fragments serving as biological connectors between bone particles. Second, the integration of this fibrin network into the regenerative site facilitates cellular migration, particularly for endothelial cells necessary for the neo-angiogenesis, vascularization, and survival of the graft. Third, the platelet cytokines (PDGF, TGF-b, IGF-1) are gradually released as the fibrin matrix is resorbed, thus creating a perpetual process of healing. Lastly, the presence of leukocytes and cytokines in the fibrin network can play a significant role in the self-regulation of inflammatory and infectious phenomena within the grafted material; PRF is also a supportive matrix for bone morphogenetic protein ${ }^{1}$.

With all these advantages, PRF comes with one major drawback of silica impregnation caused due to the activation of the blood clot, with the help of the glass tubes. T-PRF tries to overcome this disadvantage by using the titanium tubes for activating the clot. In their initial trials, Tunali et al. found that, although titanium induced platelet aggregation similar to that in glass tubes, and the clot produced in the titanium was clinically identical to that produced in the glass tubes, the activation of platelets with titanium induces distinctive characteristics of T-PRF, including its increased biocompatibility².

Aim

The aim of this study is to evaluate effects of the autogenous platelet concentrates such as L-PRF and T-PRF, and compare these effects with xenografts on early bone healing.

Materials and Methods

Two bilateral tibial defects with $5 \mathrm{~mm}$ wide, $10 \mathrm{~mm}$ long, $3 \mathrm{~mm}$ depth were constituted on 20 male rabbits. The blood samples were centrifuged at $2800 \mathrm{rpm}$ for $15 \mathrm{~min}$. In group 1, at right legs, first defect was filled the anorganic bovine bone (ABB) and L-PRF, second defect was filled only L-PRF. In group 2, first defect was filled the ABB and T-PRF, second defect was filled only T-PRF. In both groups at left legs, ABB and collagen membrane were used in first defect, second defect was untreated as control. Half of the animals from each group were sacrificed after 7 days and the remaining animals were sacrificed after 21 days. All legs were amputated for histopathological and immunohistochemical evaluations. New bone formation, fibrosis and the amount of the residual grafts were evaluated histopathological. Immunohistochemical examinations of the expression of BMP-2 and FGF-2 were performed.

\section{Results}

According to the results of this study, it is found that collagen membrane + xenograft group had statistically significant lower scores than the other groups in the histopathologic examination and showed the formation of new bone, there is no statistically significant difference between the other groups.

It is observed that more fibrosis occurred in L-PRF graft (-) and L-PRF graft $(+)$ groups than the other groups in the seventh and twenty-first days. L-PRF produced significantly more fibrosis when used alone than used with bone graft as a membrane. Despite L-PRF groups, TPRF groups with graft or alone caused the least amount of fibrosis in the seventh and twenty-first days. There was no significant difference in the amount of residual grafts between the studied groups. It is found that the amount of residual grafts in the twenty-first days are less than the seventh days for all groups. In according to results of immunohistochemical analysis, it is found that there is no statistically significant difference between the expressions of BMP-2 and FGF-2 in all groups.

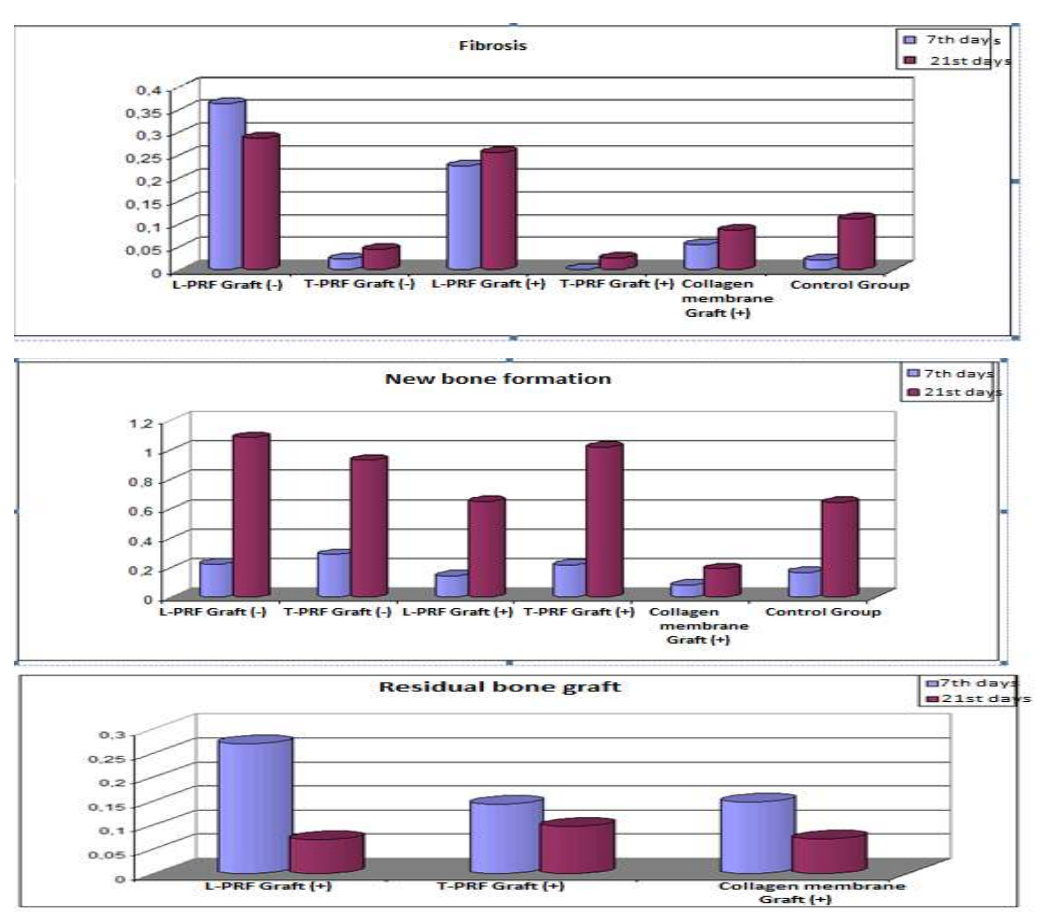

\section{Conclusions}

The use of platelet concentrates are becoming widespread in improving of the bone regeneration. Obtaining the tube from different materials, addition of activator material into the tube, placement angle of the tube, the change of the time, direction and centrifugation speed make a difference in the histological character of the fibrin and its release of growth factor and resorption time. T- PRF causes less soft tissue in the operation area than L-PRF. There is no significant difference between the L-PRF and T-PRF in graft resorbtion time.

1) Choukroun J, Adda F, Schoeffler C, Vervelle A. Une opportunit_e en paroimplantologie: le PRF. Implantodontie 2001; 42: 55-62.

2)Tunali M, Ozdemir H, Kucukodacı Z, Akman S, Yaprak E, Toker H, Firatli E. A novel platelet concentrate: Titaniumprepared platelet-rich fibrin. BioMed Research International; 2014.

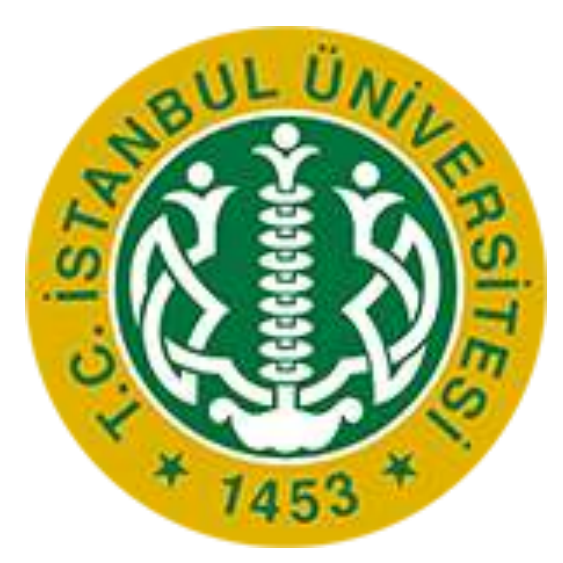

